\title{
THE CORRELATION OF FAT MASS AND FAT FREE MASS WITH PEAK EXPIRATORY FLOW RATE IN MEDICAL STUDENTS OF GOVERNMENT MEDICAL COLLEGE, THRISSUR
}

\author{
Noopura Babu P. M11, Mini K²
}

1 Junior Resident, Department of Physiology, Government Medical College, Thrissur. ${ }^{2}$ Additional Professor, Department of Physiology, Government Medical College, Thrissur.

\section{ABSTRACT}

\section{BACKGROUND}

Obesity and its strong association with ventilator dysfunction have increased the scope of studies related to obesity and pulmonary function tests. There are many studies conducted in different parts of the world, which have reported that increased body weight decreases lung volume. But majority of studies are conducted based on the BMI. The major limitation of BMI is that it does not take into account the actual composition of bodyweight. Excess body weight may be due to adipose tissue or muscle hypertrophy, both of which will be considered as excess mass. A deficit of BMI may be due to decreased amount of adipose tissue Fat mass (FM) or Fat Free Mass (FFM) deficit.

In the present study, we are trying to study the correlation between FM, FFM and PEFR in the Ist MBBS students of Medical College, Thrissur.

\section{MATERIALS AND METHODS}

The $1^{\text {st }}$ MBBS students of Government Medical College, Thrissur, from February 2014 to February 2015 were taken as the study population. 104 students which included 54 boys and 50 girls were selected by random sampling method. Their anthropometric measurements, PEFR and fat mass assessed. Fat mass measured using Body mass analyser, which utilises the principle of bioimpedance to calculate the parameter. A portable electronic spirometer (MIR SPIROBANK) is used to assess PEFR. Sample size calculation was done based on a study by Santana $\mathrm{H}$ et al in 2001. Sample size calculated was 80.

\section{RESULTS}

Mean age of the study participants was $19.64+$ or -0.835 years. Mean BMI was 21.5 and $98 \%$ of study population belong to normal range. Fat mass of females was found to be significantly higher than that of males. Body composition, fat mass show significant negative correlation with PEFR in males. In males, FFM has significant positive correlation with PEFR also.

\section{CONCLUSION}

Body composition is a significant determinant of lung function, namely PEFR. The current study suggests negative correlation of fat mass and positive correlation of fat free mass with PEFR. Additional studies may be required in order to use these factors for pulmonary function prediction formula. Not only age, gender, height and weight, but body fat percentage and muscle mass also determine pulmonary function such as PEFR of an individual.

\section{KEYWORDS}

Fat Mass, Fat Free Mass, Peak Expiratory Flow Rate, Cross Sectional Study.

HOW TO CITE THIS ARTICLE: Babu NPM, Mini K. The correlation of fat mass and fat free mass with peak expiratory flow rate in medical students of Government Medical College, Thrissur. J. Evolution Med. Dent. Sci. 2017;6(33):2730-2734, D0I: $10.14260 /$ Jemds/2017/589

\section{BACKGROUND}

The dramatic increase in the prevalence of obesity and its strong association with ventilator dysfunction has raised the need of studies related to obesity and pulmonary function tests. Obese people are at an increased risk of respiratory symptoms such as breathlessness, especially while doing exercise, even if they have no obvious respiratory illness..$^{1,2}$ Obese patients have decreased exercise capacity and increased dyspnoea, which influences the quality of life. ${ }^{3}$ Increased weight can cause various deleterious effects to

Financial or Other, Competing Interest: None.

Submission 13-02-2017, Peer Review 27-03-2017,

Acceptance 03-04-2017, Published 24-04-2017.

Corresponding Author:

Dr. Mini $K$,

Additional Professor

Department of Physiology,

Government Medical College, Thrissur.

E-mail: melminivasudevan @gmail.com

DOI: $10.14260 /$ jemds $/ 2017 / 589$

(c) $($ ) $($ ) respiratory function, such as decrease in respiratory muscle strength and endurance, lower control of breathing, decrease in pulmonary gas exchange, alterations in respiratory mechanics and limitations in pulmonary function tests and exercise capacity. ${ }^{4}$

There are many studies conducted in different parts of world, which have reported that increased body weight decreases lung volume. But majority of the studies are conducted based on BMI. The major limitation of BMI is that it does not take into account the actual composition of body weight. Excess body weight may be due to adipose tissue or muscle hypertrophy, both of which will be considered as 'excess mass.' On the other hand, a deficit of BMI may be due to decreased amount of adipose tissue Fat Mass (FM) or Fat Free Mass (FFM) deficit. ${ }^{5}$ Most of the earlier studies associating effect of body mass on pulmonary function have been done relating to BMI, but not FM and FFM. In the present study, we are trying to study the correlation between FM, FFM and peak expiratory flow rate in medical students of medical college, Thrissur. 
Higher BMI could be due to increased FM, FFM or both, which have been shown to have negative effects on lung function in several studies. Previous studies of the relation of body composition to lung function produced inconsistent results. At the same time, most studies have reported that FFM is positively associated with lung function, some have failed to find any association between FM and lung function, 6,7 possibly because of the limited ability of the small sample to detect a difference when the effects are modest or concentrated at particularly high amounts of FM. It has been suggested that marked degrees of adiposity may be needed for adiposity to influence pulmonary function.

Deposition of fat in the abdominal region may directly impede the descent of the diaphragm, whereas thoracic compliance and rib cage movement is diminished due to fat deposition in the chest wall and both of which lead to restrictive respiration impairment. ${ }^{8}$ At the same time very high amounts of FM and adiposity may be related to a greater degree of airway narrowing than would be expected on the basis of reduced lung volume alone, although the mechanisms remain uncertain. Most studies have shown FFM to be associated with increased lung function. Forced inspiration requires muscular contraction to overcome air pressure on the large surface area of the chest wall for forced inspiration. Greater amounts of FFM may be associated with physical activity, which tends to be associated with higher lung function.

Different expiratory flow rates are employed for demonstrating the narrowing of airways. Peak Expiratory Flow Rate (PEFR) is one such parameter. The primary factors that affect PEFR are the strength of the expiratory muscles generating the force of contraction, the elastic recoil pressure of the lungs and the airway size. In the studies conducted by Ghobain and co-workers, the obese subjects had lower PEF values than the non-obese subjects. Low PEFR in obese subjects was explained by an increase in total respiratory resistance and airway resistance with obesity.

\section{MATERIALS AND METHODS}

\section{Sampling}

Simple random sampling was used to select the participants for the study. The sample size was calculated based upon a study by Santana $\mathrm{H}$ et al $^{9}$ in 2001 , applying the formula for cross-sectional study. Correlation coefficient 0.299 and with a power of $80 \%$ sample size was calculated to be $80 ; 80$ randomly selected students who do not have any previous history of longstanding pulmonary complaints and were willing to volunteer for the study after detailed demonstration of the procedure were included in the study after getting written informed consent.

104 students of Government Medical College, Thrissur, which included ( 54 boys and 50 girls) were invited to assess their anthropometric measurements, pulmonary function and fat mass. Their general and respiratory system examinations were carried out to rule out gross abnormalities. Three subjects were allowed to perform the test in a day and they were encouraged to do the trials before taking the final readings. With the subjects wearing light indoor clothes and no shoes, body weight in kilograms was measured using a standard weighing machine and height in centimetres with a stadiometer. BMI was then calculated as body weight adjusted for stature. Then the subjects were made to sit in a chair comfortably. Their name, sex, age, height, weight and ethnicity were entered in the spirometer. After this the procedure was explained and demonstrated to them. In order to prevent the leakage of air, a mouth piece was used. Using a nose clip, subject's nose was occluded. Initially, they were instructed to take some tidal respirations. When their end tidal breath was detected by the machine, they were instructed to inhale as fast as they can to their maximum capacity. This was followed by expelling out of air completely. They were encouraged to blow out until they were unable to go on. Until three acceptable and reproducible tracings were obtained, subjects were allowed to repeat the test.

Fat mass was measured using Body Fat Analyser, which utilises the principle of bioimpedance to calculate the parameter. After entering height, weight, age and gender into the machine, the subject is asked to stand bare foot with both feet slightly apart. Subject holds the machine with his arms straight out without moving his body. Measurement is taken automatically in a few seconds.

\section{Definition of Outcome and Important Variables}

At the end of the study, it is expected that information regarding the correlation between the study variables and their significance will be brought into light.

\section{Inclusion Criteria}

$1^{\text {st }}$ MBBS Students of Government Medical College, Thrissur who do not have any previous history of longstanding pulmonary complaints.

\section{Exclusion Criteria}

Subjects who have longstanding pulmonary ailments such as bronchial asthma from childhood.

Subjects under medication.

\section{Data Collection Tools}

A portable electronic spirometer (MIR Spirobank II spirometer and Oximeter) is used for assessing pulmonary function. Standardisation of the equipment was done before starting the procedure. The MIR Spirobank II works bidirectional digital turbines for flow and volume measurements. Temperature sensor used here is the semiconductor and infrared interruption is the measurement principle. With this device two different types of turbine sensors can be used; reusable and single patient disposable. In the present study, reusable turbine was used which can be cleaned by immersing it in cold detergent solution and rinsing with cold water. A disposable mouth piece was used to connect the subject to the spirometer. Body weight in kilograms was measured using a standard weighing machine and height in centimetres with a stadiometer.

For estimating the body composition, Omron Body Fat Analyser is used in the present study which works on the principle of biomechanical impedance. The electrical conductivity of fat free mass is higher than that of fat. The output is directly given as percentage of body fat. Tissues containing much water such as muscles, blood vessels and bones are highly conductive with electricity, but fat tissues are not. Therefore, by using bioimpedance principle, it is possible to determine the ratio of fat tissue compared to other tissues in the body by measuring the electrical resistance of the body tissues using extremely weak electric 
current applications to the body. The instrument is suitably calibrated. Quality checks as required by the manufacturer are followed to ensure accurate measurements.

The data was analysed using SPSS software version 18 . Analysis was done by independent sample ' $t$ ' tests, correlation and regression analysis.
RESULTS

Correlation between Body Composition and Ventilatory Function in males and females

Correlation between various body composition parameters and PFT parameters in females and males are displayed in Tables 1 and 2 respectively.

\begin{tabular}{|c|c|c|c|c|c|c|c|c|c|c|}
\hline \multirow{2}{*}{ Height } & \multicolumn{2}{c|}{ BMI } & \multicolumn{2}{c|}{ FM } & \multicolumn{2}{c|}{ FFM } & \multicolumn{2}{c|}{ FFMI } \\
\cline { 2 - 9 } & $\mathbf{r}$ & $\mathbf{p}$ & $\mathbf{r}$ & $\mathbf{p}$ & $\mathbf{r}$ & $\mathbf{p}$ & $\mathbf{r}$ & $\mathbf{p}$ & $\mathbf{r}$ & $\mathbf{p}$ \\
\hline PEFR & .445 & .001 & .038 & .794 & -.212 & .139 & .212 & .139 & .158 & .272 \\
\hline \multicolumn{3}{|c}{ Table 1. Correlation between Body Composition and Ventilatory Function in Females } \\
\hline
\end{tabular}

In females, height showed significant positive correlation PEFR. BMI showed no significant correlation with any of the lung parameters (Figure 1,2). Correlation with Fat mass and PEFR was not significant (Figure 3 \& Figure 4). FFM showed no significant correlation with PEFR (Figure 5, Figure 6).

\begin{tabular}{|c|c|c|c|c|c|c|c|c|c|c|}
\hline & \multicolumn{2}{|c|}{ Height } & \multicolumn{2}{|c|}{ BMI } & \multicolumn{2}{|c|}{ FM } & \multicolumn{2}{|c|}{ FFM } & \multicolumn{2}{|c|}{ FFMI } \\
\hline & $r$ & $\mathrm{p}$ & $r$ & $\mathrm{p}$ & $r$ & p & $r$ & $p$ & $r$ & $\mathrm{p}$ \\
\hline PEFR & .484 & .000 & -.072 & .604 & -.271 & .048 & .271 & .048 & .174 & .208 \\
\hline
\end{tabular}

Among males, height showed significant positive correlation with PEFR. BMI showed no significant correlation with any of the lung parameters (Figure 1 \& Figure 2). But the body composition, FM showed significant negative correlation with PEFR (Figure 3, Figure 4). FFM showed significant positive correlation with PEFR (Figure 5- Figure 6).

\section{Correlation between BMI and PEFR in Females}

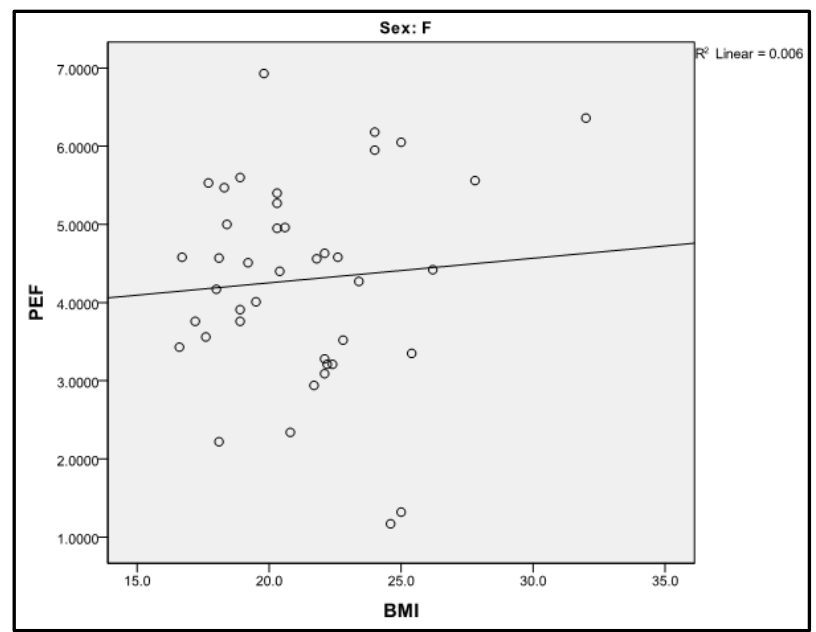

Figure 1. Relation between BMI and PEFR in Females

The correlation is positive.

Correlation coefficient ' $r$ ' is .038.

$p$ value $=0.794$ (Non-significant).

\section{Correlation between BMI and PEFR in Males}

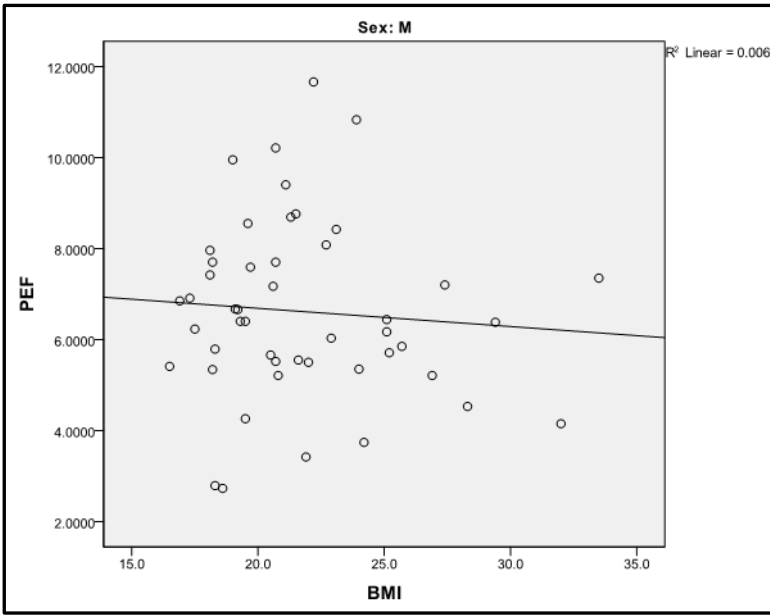

Figure 2. Relation between BMI and PEFR in Males

The correlation is negative.

Correlation coefficient ' $r$ ' is -.072 .

$\mathrm{p}$ value $=0.604$ (Non-significant).

Correlation between FM and PEFR in Females.

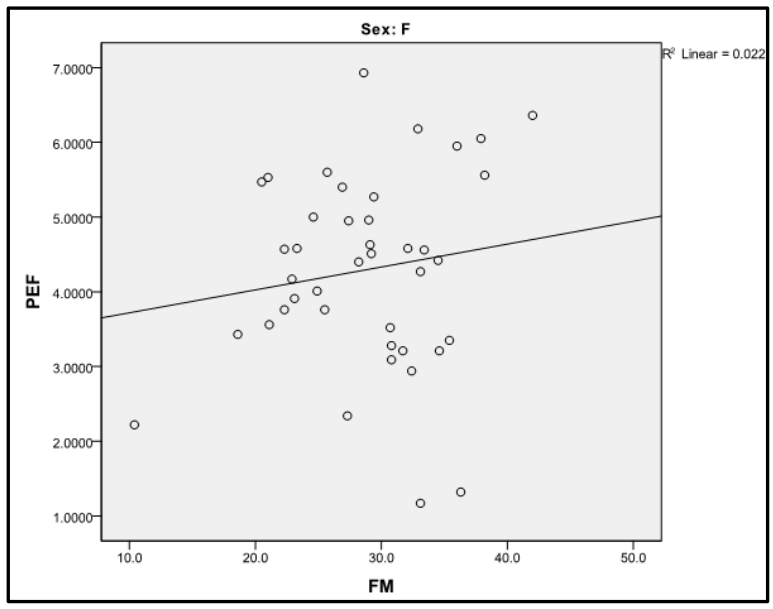

Figure 3. Relation between FM and PEFR in Females

The correlation is negative.

Correlation coefficient ' $r$ ' is -.212

$p$ value $=0.139$ (Non-significant) 


\section{Correlation between FM and PEFR in Males}

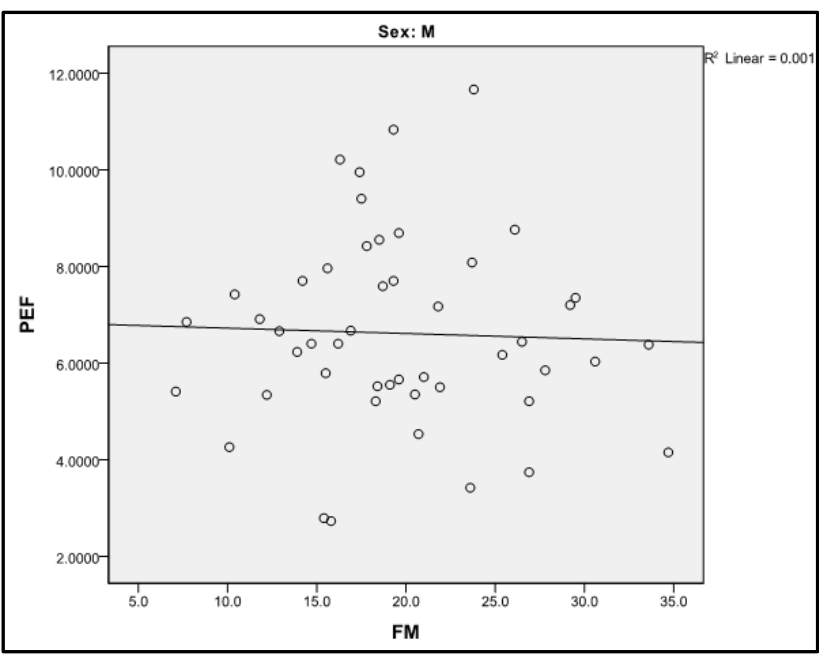

Figure 4. Relation between FM and PEFR in Males

The Correlation is Negative.

Correlation coefficient ' $r$ ' is - .271

$\mathrm{p}$ value $=0.048$ (Significant)

\section{Correlation between FFM and PEFR in Females}

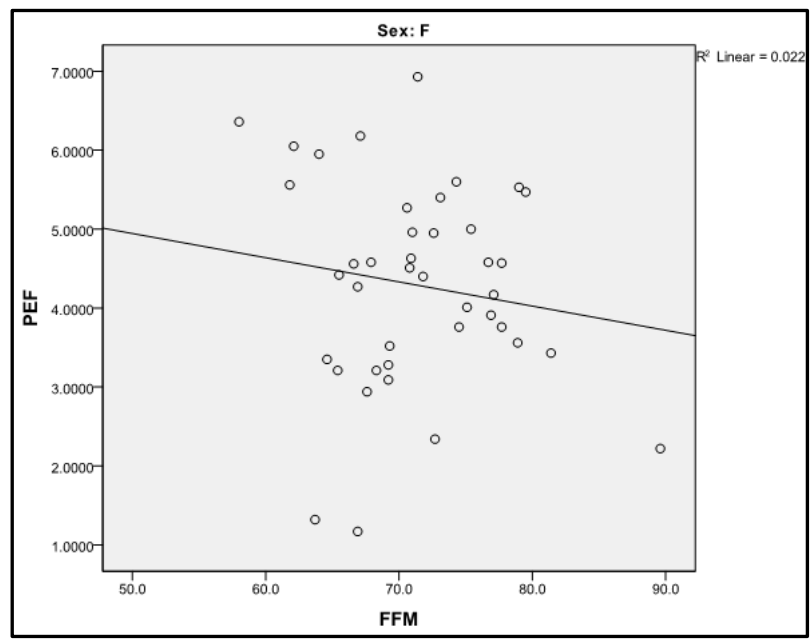

Figure 5. Relation between FFM and PEFR in Females

The Correlation is Positive.

Correlation coefficient ' $r$ ' is .212

$\mathrm{p}$ value $=0.139$ (Non-significant)

\section{Correlation between FFM and PEFR in Males}

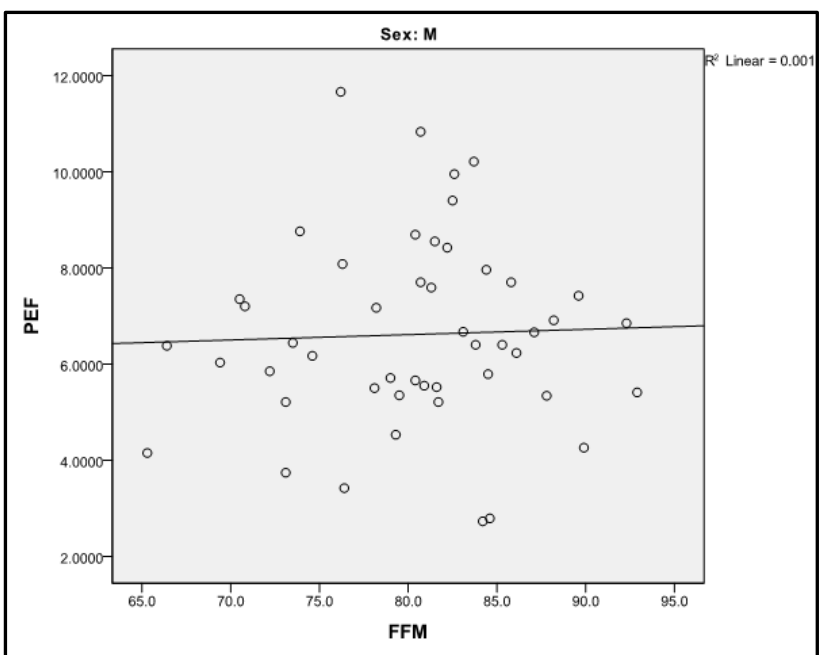

Figure 6. Relation between FFM and PEFR in Males

The correlation is positive.

Correlation coefficient ' $r$ ' is .271.

$\mathrm{p}$ value $=0.048$ (Significant) Mean age of the study population is $19.64+/-0.835$ years.

Mean BMI of the study population was found to be 21.5 and maximum number of subjects belong to normal range.

Fat mass of the females was found to be significantly higher than that of males.

Height in both males and females showed significant positive correlation with PEFR.

BMI showed no significant correlation with any of the lung parameters in both sexes.

Body composition FM showed significant negative correlation with PEFR in males. In males, FFM has positive significant correlation with PEFR also.

\section{DISCUSSION}

\section{Relation between BMI and Ventilatory Function}

In the present study, BMI showed no significant correlation with any of the lung parameters in both sexes. The effects of BMI on spirometric values are not consistent in most of the studies, some studies showing no effects ${ }^{10,11,12}$; and some other studies showing significant effects. The amount of body fat and pattern of fat distribution is related to lung function via several mechanisms, such as mechanical effects on the diaphragm which impedes the descent into the abdominal cavity and on the chest wall primarily due to the changes in compliance and in the work of breathing and the elastic recoil. ${ }^{13}$ In the present study, PEFR in males showed negative correlation with BMI which was not statistically significant. Correlation of BMI with PEFR in females is positive, but not significant. This is similar to the study done by Mohammed A G.11 Males did not show correlation of FVC and FEV 1 with BMI and body fat percentage in the study conducted by Park JE and co-workers, but showed significant correlation with FFMI, whereas females showed no significant correlation with FFMI but with BMI and body fat percentage. This was because in males most of the body weight was composed of FFM, likewise in females' body, fat percentage was higher than that of males. According to the study conducted by Jones $\mathrm{R}$ L, it was found that BMI has significant effects on all of the lung volumes, and the greatest effects were on FRC and ERV, 
which occurred at BMI values $<30 \mathrm{~kg} / \mathrm{m} .^{2}$ In the study conducted by Dharamshi and co-workers it was noted that there is a statistically significant variation in PEFR with an increase in BMI in both males and females, which is not similar with our findings.

\section{Relation between Fat Mass and Peak Expiratory Flow Rate}

Body composition FM showed significant negative correlation with PEFR in males. This association could be due to the mechanical effects of fat deposits on rib cage. Large abdominal fat mass might impede descent of the diaphragm during forced inspiration as well. Likewise, fat being a metabolically active substance, there could be metabolic effects which were not excluded in the present study. This was similar to studies conducted by Lazarus and co-workers. According to them FVC was not significantly associated with BMI, but was negatively associated with fat percentage. Low PEFR in obese subjects can be explained by an increase in total respiratory resistance and airway resistance with obesity. In the studies conducted by Ghobain and coworkers, ${ }^{11}$ the obese subjects had lower PEFR values than the non-obese subjects. Low PEFR in obese subjects was explained by an increase in total respiratory resistance and airway resistance with obesity.

Among obese women FM is distributed peripherally, whereas among obese men it is distributed more centrally. As a result, changes in FM have a greater impact on FVC in men. ${ }^{14}$

\section{Relation between Fat Free Mass and Peak Expiratory Flow Rate}

Most studies have shown FFM to be associated with increased lung function. The effects of FFM on lung function may be associated with stronger respiratory musculature. In the present study among males FFM has positive significant correlation with PEFR, whereas in females PEFR did not show significant positive association with FFM. These results are similar to the observations seen in study conducted by Pradhan B B. The difference in males and females could be due to the fact that PEFR is highly effort dependent, that the subject must initially exhale as hard as possible to obtain reproducible data. Muralidhara and co-workers ${ }^{15}$ observations did not show any correlation of PEFR with any body composition parameters, which is against our findings. In case of study conducted by Zuhal et al, PEFR values were lower in obese children than in non-obese children. In order to overcome air pressure on the large surface area of the chest wall, forced inspiration requires muscular contraction. Enright $\mathrm{P}$ et al found an association between respiratory muscle strength and ventilatory function among older subjects. This study supports the view of the studies that respiratory muscle strength has effect on respiratory function.

In this study, it was found that BMI showed no significant correlation with any of the lung parameters in both sexes. But the body composition FM showed significant negative correlation with both males and females and also with PEFR in males. In males, FFM has positive correlation with PEFR also. This helps to identify and treat these changes at an early stage in order to prevent negative effects on health and quality of life.

\section{CONCLUSION}

Body composition is a significant determinant of lung function. Current study suggests negative correlation between lung function and fat mass and a positive correlation with fat free mass, which will help in the assessment of effect of weight on lung function.

Not only age, gender, height and weight but body fat percentage and muscle mass also affect FVC, FEV 1 and PEFR. Studies involving effect of body fat distribution on ventilatory function has to be conducted. Additional studies may be required in the future in order to use these factors for a pulmonary function prediction formula.

\section{ACKNOWLEDGEMENT}

I thank Dr. K. P. Kalyanikutty and Dr V. V. Unnikrishnan who were of constant help at all stages of the conduct of the study.

\section{REFERENCES}

[1] Bai J, Peat JK, Berry G, et al. Questionnaire items that predict Asthma and other respiratory conditions in Adults. Chest 1998;114(5):1343-8.

[2] Sahebjami H. Dyspnea in obese healthy men. Chest 1998;114(5):1373-7.

[3] Rubinstein I, Zamel N, DuBarry L, et al. Airflow limitation in morbidity obese, non-smoking men. Ann Intern Med 1990;112(11):828-32.

[4] Parameswaran K, Todd DC, Soth M. Altered respiratory physiology in obesity. Canadian Respiratoy Journal 2006;13(4):203-10.

[5] Rasslan Z, Stirbulov R, da Conceicao CAL, et al. Funcao pulmonar e obesidade. Rev Bras Clin Med 2009;7:36-9.

[6] Amara CE, Koval JJ, Paterson DH, et al. Lung function in older humans: the contribution of body composition, physical activity and smoking. Ann Human Biol 2001;28(5):522-36.

[7] Mohamed EI, Maiolo C, Iacopino L, et al. The impact of body weight components on forced spirometry in health Italians. Lung 2002;180(3):149-59.

[8] Maiolo C, Mohamed EI, Carbonelli MG. Body composition and respiratory function. Acta Diabetol 2003;40(Suppl 1):S32-8.

[9] Santana H, Zoico E, Turcato E, et al. Relation between body composition, fat distribution and lung function in elderly men. The American Journal of Clinical Nutrition 2001;73(4):827-31.

[10] Jenkins SC, Moxham J. The effects of mild obesity on lung function. Respir Med 1991;85(4):309-11.

[11] Al Ghobain M. The effect of obesity on spirometry tests among healthy non-smoking adults. BMC Pulmonary Medicine 2012;12:10.

[12] Watson RA, Pride NB. Postural changes in lung volumes and respiratory resistance in subjects with obesity. J Appl Physiol (1985) 2005;98(2):512-7.

[13] Luce JM. Respiratory complications of obesity. Chest 1980;78(4):626-31.

[14] Sue DY. Obesity and pulmonary function: more or less? Chest Journal 1997;111(4):844-5.

[15] Muralidhara DV, Bhat MR. Some aspects of pulmonary functions in the underweight \& overweight human subjects. Thai Journal of Physiological Sciences 2007;20(1):3-7. 